\title{
Heart rate alteration and cognitive efficiency
}

\author{
ALAN I. HERSHMAN and DAVID GIBSON \\ University of Calgary, Calgary, Alberta T2N 1N4, Canada
}

\begin{abstract}
The present study examined cardiovascular change in relation to the variable triad of subject activation status, complexity of mental task and secondary stimulation. Results indicated that heart rate change was significantly related to the exercise/nonexercise variable and the white noise/nonnoise variable and nonsignificantly to task difficulty. Findings revealed that heart rate was greater in the easy task than in the difficult task during the exercise condition. Interpretative inconsistencies between the Lacey position and traditional arousal theory were explored in terms of the compounding effects of internal and external "noise."
\end{abstract}

Lacey, in an examination of characteristic autonomic system patterning and motor impulsivity, concluded that ideational activity (e.g., cognitive elaboration) and noxious stimuli were both accompanied by increased cardiovascular rate, while external stimuli tended to be associated with deceleration of the cardiovascular system (Lacey, 1959; Lacey, Bateman, \& Van Lehn, 1963; Lacey \& Lacey, 1958). Lacey's claim was that heart rate (HR) level had a facilitating effect either for increased ease of environmental intake or for the rejection of the environment when internal mental activity was underway. In order to explain their findings, Lacey, Kagan, Lacey, and Moss (1963) hypothesized that (1) autonomic system change was a concomitant of both external environmental conditions and cognitive functioning, and (2) cardiac deceleration resulted in disinhibition of the cortex, facilitating intake of environmental stimuli, whereas cardiac acceleration led to central nervous system inhibition and a dampening of sensory intake. Lacey et al. (1963) contended, therefore, that the activation process is not reflective of merely the intensity level of behavior but also of the intended aim of the behavior; that is, when the organism's intended aim is to reject environmental stimuli, a cardiovascular acceleratory response is produced. Conversely, when the intended aim of the organism is to accept environmental stimulation, cardiac deceleration occurs.

While the Lacey postulates are demonstrable experimentally, they are difficult to reconcile with classical arousal theory. Consistent with Duffy's (1957) arousal construct, HR should be high during states of relative cortical activation and lower during states of relative cortical inactivation. The resulting theory implies that degree of activation is a direct function of the complexity of the task and the intensity of the stimuli, so that activation is expected to be higher during complex (difficult) tasks as opposed to more simple mental work. Malmo (1965) noted, however, that during a mental activity sequence, autonomic nervous system recordings display a progressive rise, with electroencephalogram recordings displaying constancy of cortical activity for the duration of the task. Malmo thus viewed acceleration in HR as part of the physiological functioning required to maintain a so-called "even" level of attention from onset to completion of a mental task. Malmo (1959) has further described behavioral efficiency and its relation to activation as an inverted $U$; that is, level of performance on a task rises monotonically with the increasing activation level up to a certain point, but beyond that optimal point, a further increase in the activation level contributes to performance decrements.

Other work in the area of cognitive and autonomic systems' co-functioning also suggests that Lacey's proposals are in need of reexamination. Kaufman, Gibson, and Adamowicz (1967) tested cardiovascular change in relation to cognitive effort and reported that the greatest cardiovascular increases occurred with easy mental tasks. The difficult tasks were noted to evoke lesser amounts of cardiac quickening. Adamowicz, Gibson, and Kaufman (1967) obtained similar findings using respiratory change as the index of physiological activation.

Recent developments in the quantification and analysis of HR data now permit a more exacting treatment of the problem. For example, several of the previously cited studies computed cardiovascular alteration scores using Lacey's method (Lacey et al., 1953). Brenner (1967), in discussing the measurement of $H R$, specified that due to the high variability of HR, sampling should encompass the longest period on which to base mean HR. Dahl and Spence (1971) supported Brenner's contention. The aforementioned authors criticized the Lacey et al. (1953) method of measuring HR on the basis that mean HR measures can reflect certain testing distortions (e.g., an accelerating HR response followed by a long and gradual deceleratory response), while the method of measuring momentary fluctuations will be insensitive to these.

Given the conflicting nature of previous findings and the failure of these findings to support any of the major theoretical positions, additional examination of the Lacey intake-rejection hypothesis was undertaken. The 
present experiment was designed to elaborate the Lacey formulations in relation to: (1) the effects of altered states of physiological activation on task performance, (2) the role played by task difficulty levels on HR alteration, and (3) the role of environmental stimulation on HR alteration.

\section{Method}

Subjects were 20 University of Calgary male students. Their ages ranged from 19 to 29 years and averaged 24.2 years.

The order of presentation of the "first letters" that were to evoke sentence-length association was assigned to each of the subjects randomly.

HR was continuously measured using a Grass Model 7 polygraph. The electrocardiogram (EKG) was recorded from a standard EKG Lead III position, using $2.0-\mathrm{cm}$ silver/silver chloride electrodes. The signal was fed through a Model 7P4D Tachograph preamplifier. HR recording was conducted at a paper speed of $10 \mathrm{~mm} / \mathrm{sec}$ on two channels of the Grass polygraph. The white noise $(85 \mathrm{~dB})$ was transmitted through Armaco P 322 stereophonic earphones wired so that both of the subject's ears received the same signal at the same time. Instructions were transmitted through earphones using a Phillips cassette recorder, with individual instruction given for each condition. The subject was in a lighted sound-attenuated chamber containing a comfortable arm chair, headphones, and a pushbutton device that signaled the experimenter that the task was completed. Timing and recording equipment were located in an adjacent compartment.

A 2 by 2 by 2 repeated-measures design (Keppel, 1973) comprising two levels of activation (exercise, nonexercise), two levels of task complexity (easy task, difficult task), and two levels of secondary source stimulation (white noise, nonnoise) was employed. Pilot studies established that HR at the 135to 145 -beat per min level does not drop as rapidly as at lower levels of cardiovascular acceleration, but that beyond this level, the data become greatly distorted. Task difficulty levels were defined by establishing comparative difficulty of "first letters" to evoke sentence-length associations. Stimulus letters were then rank ordered from easiest to most difficult. Finally, $85 \mathrm{~dB}$ of white noise was found to facilitate the needed distractive element (secondary source stimulation) yet not be rated as noxious or painful.

The subjects were familiarized with the polygraph and assured that no shock or other unpleasant stimulation would be used. Subsequent to calibration, a stabilization period of 15 min ensued. This length of time was sufficient for the subject to achieve physiological stability. At the culmination of the stabilization period, cardiovascular rate was recorded for $8 \mathrm{~min}$, thus establishing the basal HR. Subjects were then assigned a letter (predetermined for that condition) with which they were to construct a grammatically correct sentence. If the condition necessitated a higher level of cardiovascular functioning, the exercise instructions were presented before the subject received the letter with which he was to form the sentence.

\section{RESULTS}

Analysis of variance of the data showed HR to vary significantly as a function of exercise/nonexercise $[\mathrm{F}(1,19)=126.35, \mathrm{p}<.001]$. The exercise condition was shown to produce the required effect; that is, HR was significantly accelerated in comparison to the nonexercise variable. These simple effects are shown in Figure 1.

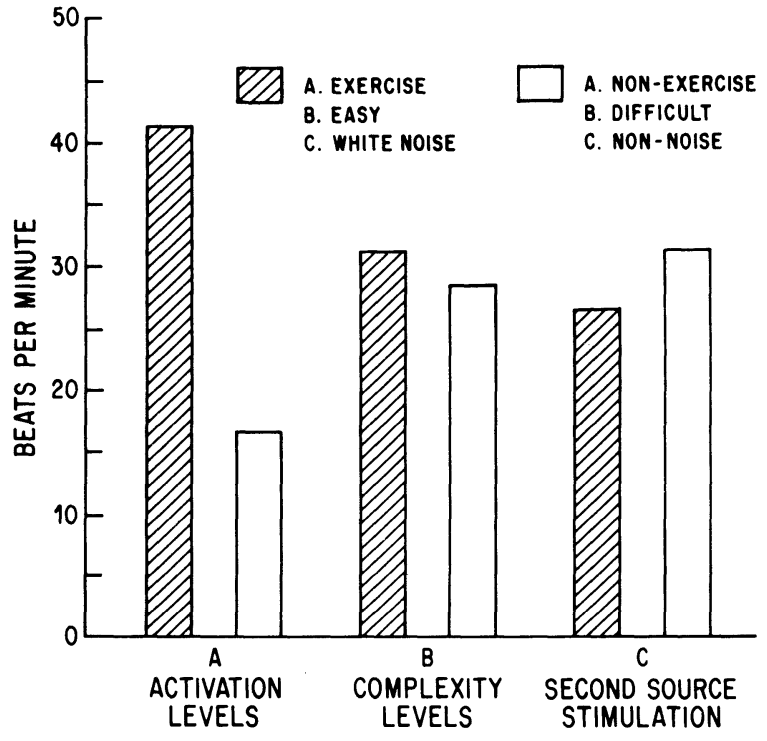

Figure 1. Comparison of heart rate simple effects for two levels of activation, task complexity, and second-source stimulation.

HR change was significantly related to the white noise/nonnoise variable $[F(1,19)=5.21, p<.05]$. Results indicate that white noise significantly decelerated $\mathrm{HR}$ in comparison to nonnoise conditions. Effects of task difficulty were not found to be significantly related to $\mathrm{HR}$ alteration $[\mathrm{F}(1,19)=2.94, \mathrm{p}>.05]$. HR was found to vary significantly as a function of the interaction between the exercise/nonexercise and task difficulty variables $[F(1,19)=8.78, p<.01]$ and the exercise/nonexercise and white noise/nonnoise variables $[F(1,19)=9.25, p<.01]$. Nonsignificant differences were observed for the interaction of task difficulty and white noise/nonnoise variables $[\mathrm{F}(1,19)=3.70, \mathrm{p}>$ $.05]$.

In order to clarify the nature of the significant interactions between (1) easy and difficult tasks during the exercise and nonexercise conditions and (2) white noise and nonnoise levels during the exercise and nonexercise conditions, correlated t tests were performed on the data. Analysis of the interactions revealed HR to be greater in the easy task in comparison to the difficult task during the exercise condition $[\mathrm{t}(19)=2.68$, $\mathrm{p}<.05]$. HR was significantly lower for the white noise condition than for the nonnoise condition during the exercise segment $[\mathrm{t}(19)=3.11, \mathrm{p}<.01]$. In the nonexercise condition, differences in HR were nonsignificant when comparisons were made between tasks involving noise and nonnoise [ $\mathrm{t}(19)=.11, \mathrm{p}>.05]$.

An analysis of variance for task duration indicated duration to solution to be related significantly to the exercise/nonexercise variable $[\mathrm{F}(1,19)=4.64, \mathrm{p}<.05]$, task difficulty $[\mathrm{F}(1,19)=19.47, \mathrm{p}<.01]$, and the white noise/nonnoise variables $[\mathrm{F}(1,19)=11.89, \mathrm{p}<.01]$. 
Duration to solution was longer for subjects functioning under the exercise condition in comparison to the nonexercise condition. Difficult tasks required a longer period to solution than the easy tasks. Subjects in the white noise conditions required longer periods of time in order to complete the required task than in the nonnoise conditions. Further, tasks involving white noise were found to have significantly greater duration to solution than nonnoise tasks in the exercise condition $[\mathrm{t}(19)=3.46, \mathrm{p}<.01]$, but not in the nonexercise condition $[\mathrm{t}(19)=1.38, \mathrm{p}>.05]$. Direction of findings is depicted in Figure 2.

The overall results are not surprising insofar as they support the Lacey position that HR acceleration is associated with the performance of a mental task. An acceleratory HR response was exhibited consistently when a silent elaboration mental task was introduced. Also, second-source stimulation was found to result in cardiovascular deceleration. In all but one condition (nonexercise, difficult task), mean HR was greater in the absence of white noise, lending support to the Lacey et al. (1953) finding that external stimulation results in cardiovascular deceleration. A decelerative effect attributable to white noise at an $85-\mathrm{dB}$ intensity level has also been reported by Davis, Buchwald, and Frankman (1955), Lacey et al. (1963), and Obrist (1963).

Other aspects of the findings are less consistent with the predominant theoretical positions. The following explanations, though speculative, are consistent with the present findings and suggest some future lines of investigation.

The analyses revealed that in the exercise condition,

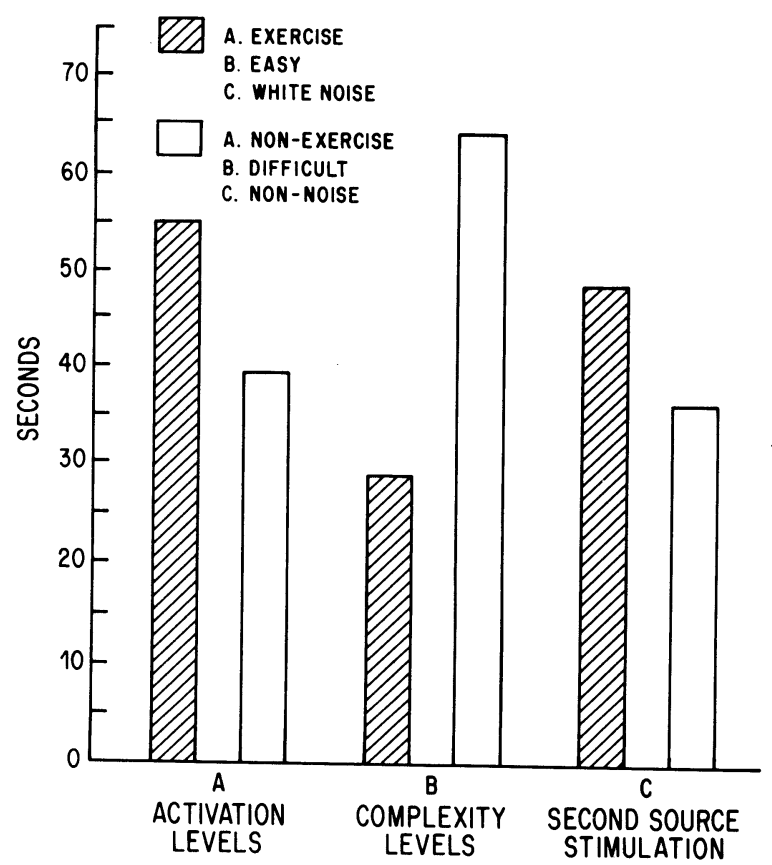

Figure 2. Comparison of task duration simple effects for two levels of activation, task complexity, and second-source stimulation. the easy task evoked significantly greater acceleration than the difficult task. In explaining this phenomenon, analysis of the requirements for the exercise condition was important. The exercise condition involved running on the spot, resulting in more pronounced breathing, elevated HR, and presumably, increased internal noise associated with the exercise. It appears, therefore, that heightened internal distraction resulted in a higher level of HR during the performance of an easy task as compared to a difficult task. Perhaps the difficult mental task invoked a sufficiently intense level of attention to satisfactorily inhibit irrelevant stimulus input without additional HR supplementation. On the other hand, an easy mental task, requiring less concentration, might require cardiac supplementation to successfully block extraneous stimulation. These results are not supportive of Duffy's (1957) conclusion that activation should be greater during complex as opposed to simple mental tasks. Lacey's (1967) postulation that HR activation should be in proportion to the need to eliminate or control distractive stimulation provides a more convincing explanation. In the absence of the exercise condition, there were nonsignificant differences between easy and difficult mental tasks. It would, therefore, seem that differing levels of HR alteration are not directly attributable to mental task difficulty levels as such. Rather, it appears that shifting levels of HR alteration, in relation to easy and difficult cognitive tasks, are facilitatory only when distracting stimulation is presented during the problem solving situation.

Comparison of the effects of white noise during the exercise and nonexercise conditions also reveals differential response patterns. The differences could be explained as follows. At the culmination of the exercise period, the subject is stimulated through two avenues of secondary source stimulation: external (i.e., white noise) and internal (i.e., noise attributable to an accelerated HR and intensified breathing). If an additive property of internal and external secondary source stimulation is postulated, such that these two sources of secondary stimulation have a greater effect than either one singly, then it would seem reasonable to expect a larger deceleratory effect during exercise than during the nonexercise condition. The results support such a position. The presence of both sources of secondary source stimulation during the exercise condition resulted in significantly lower levels of HR than for the conditions of exercise that did not employ white noise stimulation. Conversely, under the noisefree condition, nonsignificant differences were noted.

Comparisons of task duration for the individual cells under the exercise and nonexercise conditions revealed that task duration was longer under the exercise condition than under the nonexercise condition. These findings can be explained as follows. If an inverted-U relationship between $\mathrm{HR}$ activation and cognitive efficiency is postulated, it would seem that HR has been activated beyond the optimal limit for these particular 
cognitive tasks, resulting in a decrement of cognitive efficiency. Such findings have been previously reported by Malmo (1959). A second explanation would entail an appraisal of the method by which HR acceleration was evoked. The exercise condition involved strenuous physical exercise. One result of the exercise condition was a higher level of HR in conjunction with the internal noise associated with a more activated level of cardiovascular activity. Also, the subjects' attempts to take in oxygen quickly resulted in intensified visceral noise. Possibly, the accelerated HR could not compensate for both the internal and external noise stimulation, resulting in a higher level of distraction for the subject and a loss of cognitive efficiency.

In conclusion, the Lacey formulation with respect to autonomic change and mental task activity is generally supported, conditional on the subject's cardiac activity level. The results do not substantiate Lacey's findings that an accelerated cardiovascular system tends to minimize environmental intake under all circumstances, as can be evidenced by the loss of cognitive efficiency during the exercise condition in the presence of secondary source stimulation. On the basis of the present findings, there are elements of support for both the Lacey position and traditional arousal constructs. The challenge to further study will be to establish normative data along the dimensions of cognitive task demand (e.g., variety of problems presented, difficulty levels), extraneous stimuli (e.g., external and/or internal), and activation states.

\section{REFERENCES}

Adamowicz, J. K., Gibson, D., \& Kaufman, D. Respiration change and mental task. Psychonomic Science, 1967, 9, 183-184.
Brenner, J. Heart rate. In P. H. Venables \& I. Martin (Eds.), A manual of psychophysiological methods. New York: Wiley, 1967.

Dahl, D., \& Spence, P. Mean heart rate predicted by task demand characteristics. Psychophysiology, 1971, 7, 369-375.

Davis, R. R., Buchwald, A. M., \& Frankman, R. S. Autonomic and muscular responses and their relation to simple sitmuli. Psychological Monographs, 1955, 20, 1-71.

DuFFY, E. The physiological significance of the concept of "arousal" or "activation." Psychological Review, 1957, 64, 265-275.

Kaufman, D., Gibson, D., \& Adamowicz, J. D. Heart rate change under variable auditory and mental task conditions. Psychonomic Science, 1967, 9, 471-472.

KEPPEL, G. Design and analysis: $A$ researchers handbook. Englewood Cliffs, N.J: Prentice Hall, 1973.

LACEY, J. I. Psychophysiological approaches to the evaluation of psychotherapeutic process and outcome. In E. A. Rubinstein \& M. B. Parloff (Eds.), Research in psychotherapy. Washington, D.C: American Psychological Association, 1959.

LACEY, J. I. Somatic response patterning and stress: Some revision of activation theory. In M. A. Appley \& R. Trumbull (Eds.), Psychological stress: Issues in research. New York: Appleton-Century-Crofts, 1967.

Lacey, J. I., Bateman, D. E., \& Van Lehn, R. Autonomic response specificity. Psychosomatic Medicine, 1953, 15, 8-21.

Lacey, J. I., Kagan, J., Lacey, B. C., \& Moss, H. A. The visceral level: Situational determinants and behavioral correlates of autonomic response patterns. In P. H. Knaff (Ed.), Expression of the emotions in man. New York: International Universities Press, 1963.

LACEY, J. I., \& LACEY, B. C. Verification and extension of the principle of autonomic response-stereotype. American Journal of Psychology, 1958, 71, 50-73.

Malmo, R. B. Activation: A neuropsychological dimension. Psychological Review, 1959, 66, 367-386.

Malmo, R. B. Physiological gradients and behavior. Psychological Bulletin, 1965, 64, 225-234.

OвRIST, P. A. Cardiovascular differentiation of sensory stimuli. Psychosomatic Medicine, 1963, 25, 450-459.

(Received for publication April 19, 1979.) 